\title{
金属与塑料激光连接的研究现状与展望*
}

檀财旺 ${ }^{1,2}$ 苏健晖 ${ }^{1}$ 冯紫微 ${ }^{2}$ 陈 波 ${ }^{2}$ 宋晓国 ${ }^{1,2}$ 冯吉才 ${ }^{1,2}$

(1. 哈尔滨工业大学先进焊接与连接国家重点实验室 哈尔滨 150001;

2. 哈尔滨工业大学(威海)山东省特种焊接技术重点实验室 威海 264209)

\begin{abstract}
摘要: 总结了近年来金属与塑料激光连接的国内外研究成果, 分别从工艺研究、温度场模拟、气泡产生及抑制和接头性能调 控等四个方面分析了金属与塑料激光连接的难点及存在的主要问题。首先对金属与塑料的连接工艺进行讨论, 阐明激光功率、 焊接速度和离焦量等参数对接头强度的影响, 并介绍了工艺参数的优化方法; 概括了有限元温度场模拟计算对接头宽度和强 度的预测效果; 通过总结气泡的产生过程，阐述了其对接头性能的影响; 并从金属与塑料的连接机理角度阐述了接头性能调 控的手段。最后对金属与塑料的研究目前仍存在的问题进行总结, 并对其发展做出展望。
\end{abstract}

关键词: 金属/塑料; 激光连接; 温度场模拟; 气泡; 性能调控

中图分类号: TG456

\section{Research Status and Development on Laser Joining of Metal to Plastic}

\section{TAN Caiwang $^{1,2}$ SU Jianhui ${ }^{1} \quad$ FENG Ziwei $^{2}$ CHEN Bo ${ }^{2}$ SONG Xiaoguo ${ }^{1,2}$ FENG Jicai ${ }^{1,2}$}

(1. State Key Laboratory of Advanced Welding and Joining, Harbin Institute of Technology, Harbin 150001;

2. Shandong Provincial Key Laboratory of Special Welding Technology,

Harbin Institute of Technology at Weihai, Weihai 264209)

\begin{abstract}
The studies on the laser joining of metal to plastic in recent years are summarized, and the problems as well as difficulties in the laser joining process are analyzed. The research status is discussed from the following aspects: joining characteristics, simulation of temperature field at the interface, generation and suppression of gas bubbles and regulation of joint performance. In the discussion of the process, the effect of laser power, welding speed and defocus distance on the strength of joints is concluded, and the optimization methods of process parameters are introduced. The temperature field simulation calculation of finite element model on the joint width and strength is then summarized. The influence of gas bubbles on the performance of joints is stated after concluding the formation of bubbles. The way to regulate the joint strength is summarized according to the joining mechanism for metal and plastic. The potential problems are pointed out, and the conclusions and prospects for laser joining of metal to plastic are finally disclosed.
\end{abstract}

Key words: metal and plastic; laser joining; simulation of temperature field; gas bubbles; performance regulation

\section{0 前言}

近年来，随着汽车、航天工业等行业轻量化的 不断发展，轻质高强合金及工程复合材料因其质轻 且具有较高的强度及耐蚀性等优异性能广泛应用于 工业领域 ${ }^{[1-4]}$ 。由于航空工业及机车行业对于部件结 构及材料使用性能的严格要求, 实现金属与复合材 料的可靠连接已成为国内外研究的热点 ${ }^{[5]}$ 。如宝马 i8 系列新能源汽车采用铝合金框架与碳纤维复合材

* 国家自然科学基金面上(51875129)和国家高速列车技术创新中心研发计 划(CXKY-02-02(2019))资助项目。20190830 收到初稿, 20191014 收到修 改稿
料底盘相连接的设计结构使车体减重 $53 \%$ 以上，同 时能耗可降低 $50 \%{ }^{[6]}$, 波音公司部分飞机的机翼也 采用钛合金蒙皮与碳纤维增强塑料制成的翼缘的连 接结构以达到轻量化效果等。

工程塑料因其具有高韧性、低成本及与金属相 近的加工特性, 且克服了传统热固性塑料损伤容限 低、吸湿及使用期短等缺点, 近年来发展速度非常 快，广泛应用于工业领域 ${ }^{[7]}$ 。其与工业用轻质合金 通过合理的设计工艺达到原子间的结合，既可以保 证构件强度、达到轻量化的目的，又可以克服两种 材料的缺点, 拓宽其应用领域 ${ }^{[8-9]}$ 。

但是, 工程塑料的熔点一般低于 $400{ }^{\circ} \mathrm{C}$, 远低 于金属材料熔点，且塑性转变区间及热膨胀系数等 
物理性能差异很大, 形成良好接头较困难 ${ }^{[10]}$ 。普通 机械连接方法由于受到结构及材料限制, 通常密封 性较差, 且大面积部件无法形成可靠连接 ${ }^{[11]}$ 。胶接 受服役环境影响较大, 且胶质随时间延长易发生老 化 ${ }^{[12]}$ 。传统焊接方法受工艺及接头形式限制, 易产生 较大缺陷且连接强度一般较低。如电阻点焊需克服材 料导电性问题, 且连接界面不连续, 难以完成大面积 连接 ${ }^{[13]}$; 钎焊界面夹杂物较多, 同时需克服钎料对塑 料的润湿性问题 ${ }^{[14]}$; 超声波焊会破坏复合材料内部纤 维结构, 降低接头连接强度 ${ }^{[15]}$; 搅拌摩擦焊需依靠刚 度较大的摚拌头, 会产生孔洞等缺陷 ${ }^{[16]}$ 。

激光焊接是目前发展较快, 前景广阔的焊接方 法, 可以实现异种材料的高质量连接, 相比于其他 连接工艺, 其焊接缺陷较少、效率高、能适用于多 种接头形式 ${ }^{[3,17-19]}$ 。本文将从工艺研究、连接界面 温度场仿真模拟、界面位置气泡产生及抑制和接头 性能调控四个方面介绍金属/塑料激光连接技术在 国内外的发展现状, 并进一步分析其未来发展方向。

\section{1 工艺研究}

金属与塑料的激光连接工艺研究部分, 国内外主 要针对不锈钢、镁合金、铝合金以及钛合金与塑料(包 括高分子聚合物和纤维增强复合材料)进行了研究。根 据激光辐照方式不同分为激光透射焊和热传导焊。金 属侧在上为热传导焊, 塑料侧在上为透射焊, 其中, 激光透射焊只适用于金属与透明塑料的焊接。

最初开展的是金属(不锈钢、镁合金等)与聚合 物聚对苯二甲酸乙二醇酯(Polyethylene terephthalate, PET)及聚甲基丙烯酸甲酯(Polymethyl methacrylate, PMMA)等的激光连接。2008 年, 日本大阪大学 KATAYAMA 等 ${ }^{[10]}$ 利用二极管激光器对 304 不锈钢 与 PET 聚合物进行激光透射连接, 如图 1 所示 ${ }^{[10]}$, 获得了 $3000 \mathrm{~N}$ 拉剪力的接头, 首先实现了金属与 塑料的激光连接。在此基础上, 江苏大学郑怀中 ${ }^{[20]}$ 利用激光透射焊与热导焊对 304 不锈钢与 PET 分别 进行连接, 并研究了在激光连接过程中所用的激光 功率、焊接速度以及离焦量对接头强度的影响规律, 发现随着三者的增加, 接头强度均出现先增加后降 低的趋势。图 2 为不同激光功率作用下 PET/304 不 锈钢激光透射焊接头形貌 ${ }^{[20]}$, 当激光功率较低时, 焊接温度较低, PET 局部熔化与 304 不锈钢板可实 现可靠连接, 而当激光功率过大, 焊缝温度过高, PET 发生烧损, 难以实现可靠的接头。当采用热导 焊时, 相同焊接工艺参数下连接界面处温度较低, 焊缝成形良好, 接头强度达到 PET 母材的 39\%。大
连理工大学孙雪会 ${ }^{[21]}$ 对 AZ31B 镁合金与 PET 的激 光焊接模式进行了研究。作者分别采用脉冲与连续 两种激光模式对镁合金与塑料进行焊接, 同时研究 了功率密度、焊接速度及离焦量对接头性能的影响, 所获接头强度同样得出先增加后降低的趋势。且在 试验中发现, 相较于脉冲激光, 连续激光的能量密 度分布均匀, 获得了较高质量的焊接接头。

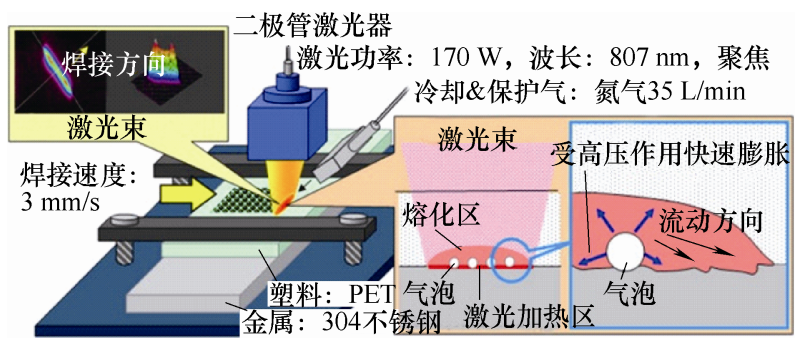

图 1 金属/塑料激光透射连接示意图

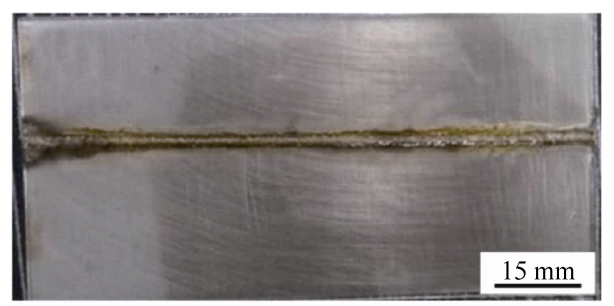

(a) 激光功率为 $300 \mathrm{~W}$

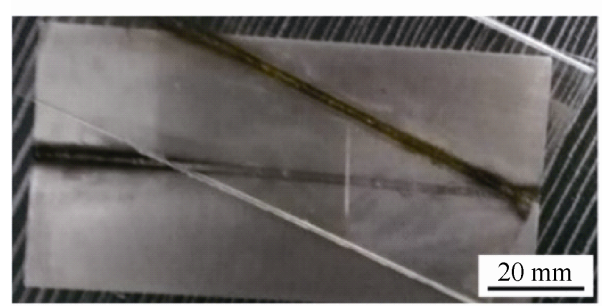

(b) 激光功率为 $400 \mathrm{~W}$

图 2 不同功率下 PET/304 不锈钢激光透射焊焊接接头

关于金属(铝合金、钛合金等)与纤维增强热塑性 复合材料(玻纤增强、碳纤增强等)激光连接的研究， 国内外开展较晚。2011 年, 德国的 ROESNER 等 ${ }^{[22]}$ 分别利用激光透射焊和热传导焊成功实现了 304 不 锈钢与尼龙(Polyamide 6, PA6)、玻璃纤维增强尼龙塑 料(Glass fiber reinforced polyamide 6, GFPA6)的连 接。日本大阪大学 JUNG 等 ${ }^{[25]}$ 利用高功率连续激光 器采用图 3 所示激光热传导连接工艺先后实现了碳 纤维增强热塑性复合材料(Continuous carbon fiber reinforced thermoplastic composites, CFRTP)与镀锌 钢 ${ }^{[23]}$ 、不锈钢 ${ }^{[24]}$ 以及铝合金 ${ }^{[25]}$ 的可靠连接, 连接接 头如图 4 所示。在三种金属与塑料的连接中, 不锈 钢与塑料的接头剪切力具有最大值 $4800 \mathrm{~N}$, 铝合金 与塑料接头具有最低剪切力为 $3000 \mathrm{~N}$, 而镀锌钢与 塑料接头的剪切力为 $3300 \mathrm{~N}$ 。进一步研究发现, 塑 料熔化区形成了亚毫米级的气泡, TEM 结果显示在 激光连接过程中，熔融塑料与金属材料表面的氧化 
膜形成中间过渡层, 并有新的化学键生成。因此能 够使得金属与塑料形成原子或分子间的紧密结合, 并最终形成可靠的接头。中国科学院宁波材料技术 与工程研究所 SHENG 等 ${ }^{[26]}$ 研究了激光功率、焊接速 度、夹持压力等激光工艺参数对 CFRTP 与不锈钢接 头性能的影响。试验发现随着热输入的增加, 接头 熔化宽度增加, 强度也逐渐提高, 而当热输入过高 时塑料分解从而降低了强度。在这些研究中, 虽然 成功实现了纤维增强复合材料与金属材料的激光连 接, 但增强纤维在连接过程中的作用机理尚不明确。

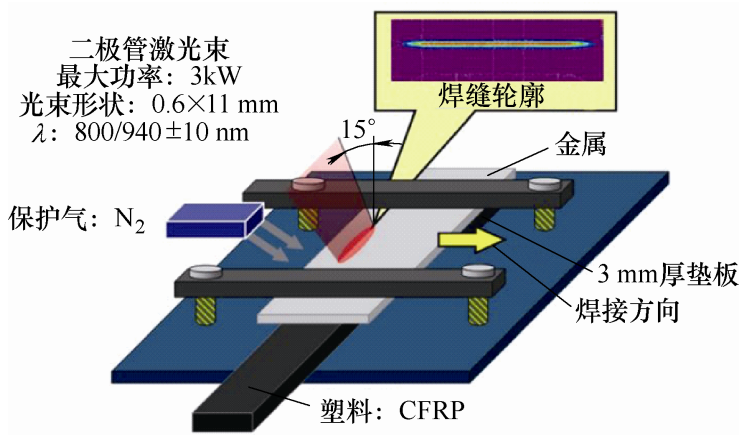

图 3 金属/塑料激光热传导连接示意图

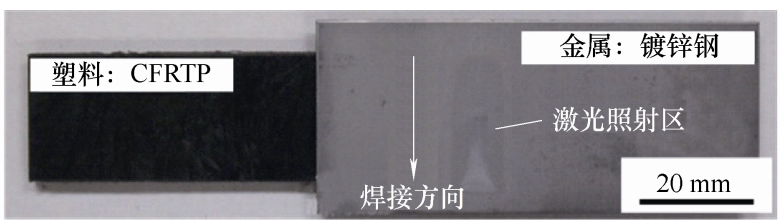

(a) 镀锌钢/CFRTP焊接接头

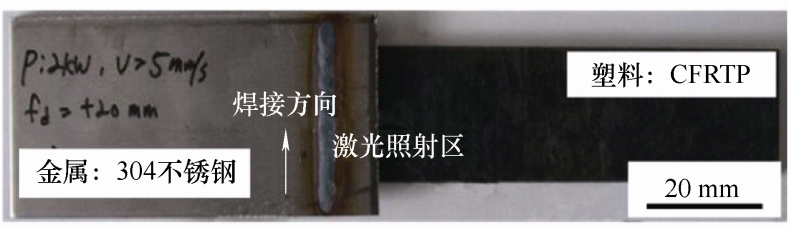

(b) 304 不锈钢/CFRTP焊接接头

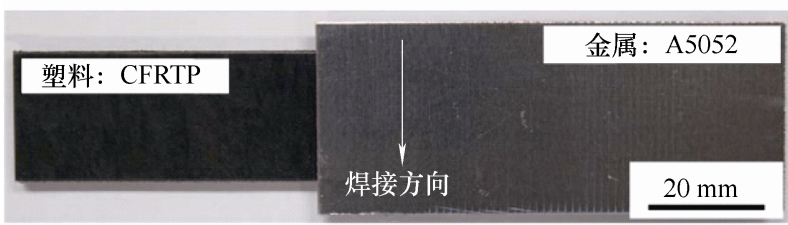

(c) A5052铝合金/CFRTP焊接接头

图 4 不同金属材料与 CFRTP 激光热导焊焊接接头形貌

笔者所在研究团队采用激光热导焊的工艺手段 也实现了碳纤维增强聚醚醚酮 (Carbon fiber reinforced poly-ether-ether-ketone, CFRP-PEEK)与钛 合金(Ti6A14V, TC4)的连接，连接接头如图 5 所示。 通过改变激光离焦量实现对焊接过程热输入的控 制, 发现随着离焦量的增加, 连接强度呈现先降低 后升高的趋势。观察如图 6 所示的 TC4/CFRP-PEEK 连接构件典型断口形貌发现, 连接界面处主要由两 部分组成: 树脂粘接区及树脂与碳纤维混合粘连的
焊缝中心区。图 7 为不同离焦量下树脂粘连区宽度 及激光扫描中心区碳纤维粘连比率变化曲线, 对比 发现随着离焦量的增加树脂连接区宽度及碳纤维粘 连比率均呈先降低后升高的趋势, 这与强度变化趋势 一致。表明改变激光离焦量会影响连接界面温度分 布, 随着离焦量的增加, 界面处树脂可熔化温度区间 宽度增加, 则树脂粘接区宽度增加, 强度随之增加。

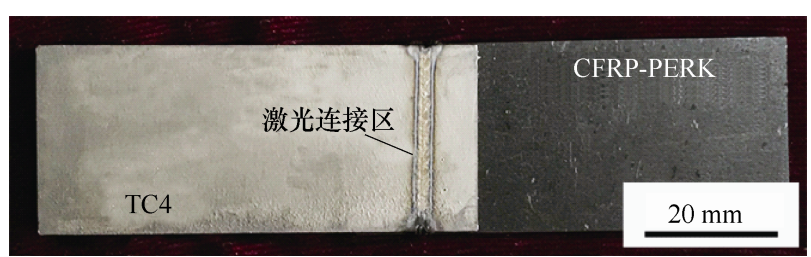

图 $5 \mathrm{TC} 4 / \mathrm{CFRP}-\mathrm{PEEK}$ 激光热导焊焊接接头形貌

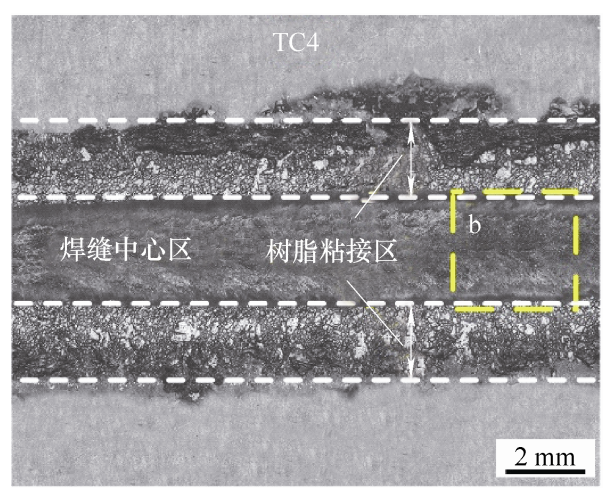

(a) 断口宏观形貌

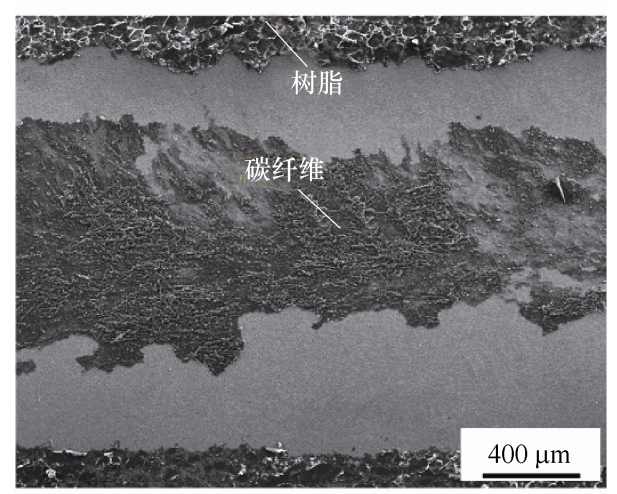

(b) 焊缝中心区局部SEM形貌

图 6 TC4/CFRP-PEEK 接头剪切断口形貌

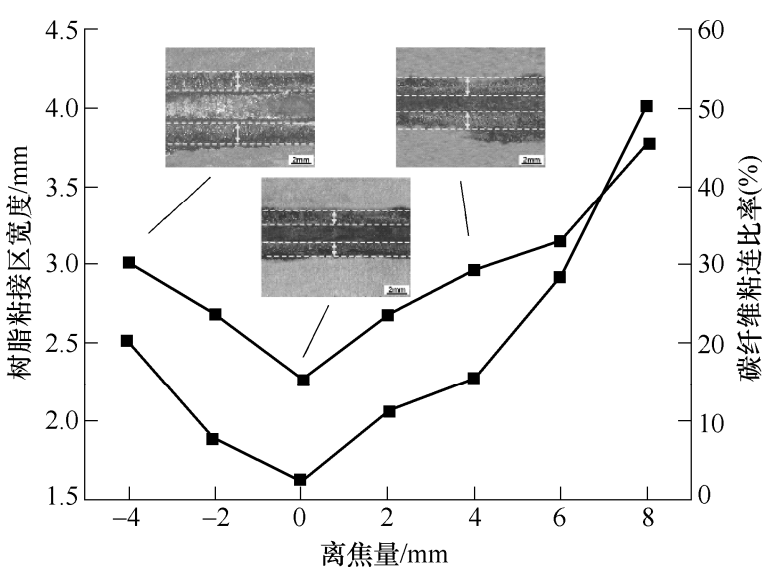

图 7 不同离焦量下树脂粘连区宽度及激光扫描中心区 碳纤维粘连比率变化曲线 
针对激光工艺参数的优化, 研究者们主要利用 响应曲面法(Response surface methodology, RSM)。 HUSSEIN 等 ${ }^{[27]}$ 利用 RSM 法分别研究了激光透射焊 与热导焊两种方法下峰值功率、脉冲持续时间、脉 冲重复率、扫描速度和脉冲波形对 304 不锈钢与 PMMA 连接的接头强度、接头宽度的影响, 图 8 为 该方法对激光扫描速度及峰值功率两重要工艺参数 对接头强度影响的预测模型 ${ }^{[27]}$, 其理论预测得到的 模型与试验中的结果吻合度较高。同时, 江苏大学 黄创 ${ }^{[28]}$ 利用半导体激光器对钛合金与碳纤维增强 尼龙(Carbon fiber reinforced polyamide66, CFPA66) 进行了连接, 建立了激光功率、离焦量及扫描速度 对接头强度与接头宽度的数学模型, 对试验结果起 到了一定的解释和预测作用。

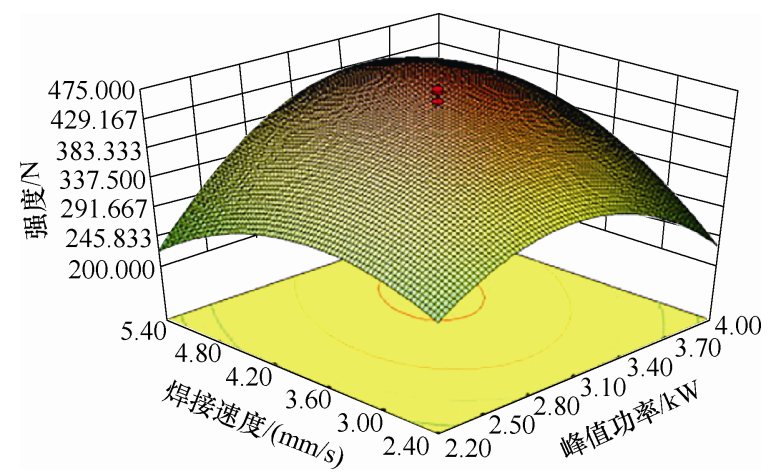

图 8 激光扫描速度及峰值功率对接头强度 的交互影响预测模型

此外, 广东工业大学黄怡洁 ${ }^{[29]}$ 研究了 304 不锈钢 与 PMMA 激光透射焊过程中激光功率、焊接速度、 光斑大小、脉冲频率等加工参数对焊缝宽度和剪切应 力的影响规律, 提出采用正交设计试验的方法, 建立 了 BP-ANN 神经网络模型算法对各工艺参数的作用 进行优化。经对比, 其建立的模型具有良好的预测能 力, 可实现最大相对误差 $11.7 \%$, 精度处于 $12 \%$ 以内。

\section{2 温度场模拟}

金属与塑料的激光连接是一个比较复杂的过程, 需要考虑激光能量的热输入、热传导、材料受热熔化 与冷却等现象, 涉及到材料学、力学以及热学等多个 学科的交叉, 是一个非线性过程。目前国内外对金属 与塑料激光连接的仿真模拟主要是利用有限元软件 建立连接温度场模型并进行规律探索和结果预测。

为了揭示接头形成与连接温度场的内在联系, 江苏大学黄创 ${ }^{[28]}$ 利用 ANSYS 有限元分析软件, 对 钛合金与碳纤维增强尼龙(CFPA66)的激光热传导 连接过程进行了温度场的模拟, 分析了不同工艺参 数包括激光功率、扫描速度以及离焦量对温度场的
影响, 从而对接头宽度进行了预测。模拟结果显示, 接头宽度随着激光功率的增加而增大, 随着扫描速 度的增加而减小，随着离焦量的增加出现先增加后 降低的趋势, 这与试验结果具有较好的拟合度。学 者 HUSSEIN 等 ${ }^{[30]}$ 和广东工业大学黄怡洁 ${ }^{[29]}$ 在对 304 不锈钢与 PMMA 进行激光透射连接时建立了温 度场模型。其中, 图 9 是 HUSSEIN 建立的温度场 模型 ${ }^{[30]}$ ，作者对其热过程进行了模拟，在一定程度 上揭示了接头的形成机理。宁波大学王强 ${ }^{[31]}$ 也对 304 与 CFRTP 的激光热传导连接温度场进行了仿真 模拟。笔者所在研究团队也采用相同热源模型实现 了对不同离焦量下 TC4/CFRP-PEEK 焊接过程的模 拟，界面连接处温度分布及不同离焦量下塑料表面 温度分布曲线如图 10 所示。通过对比不同离焦量下 CFRP-PEEK 界面温度曲线发现, 介于树脂可熔化温 度区间的宽度与实际接头中界面连接区宽度变化趋 势及强度变化趋势相同，表明该温度场模拟的计算 结果可实现对构件强度的预测。

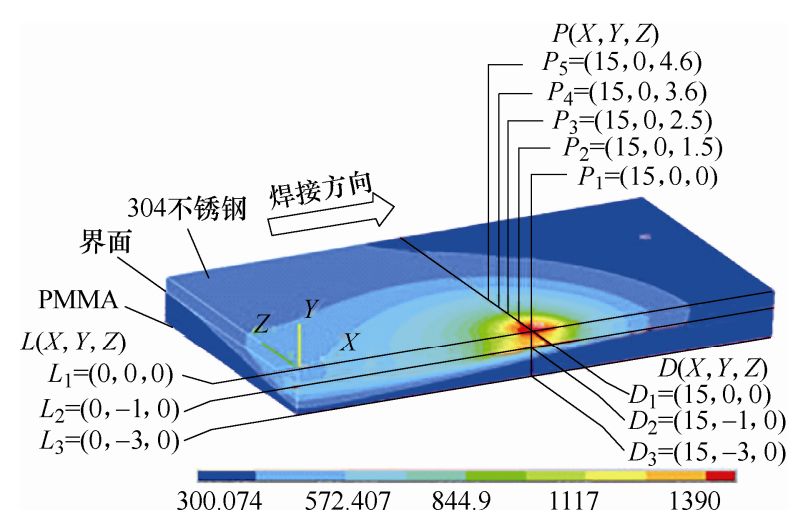

图 9 激光透射连接过程温度场模型

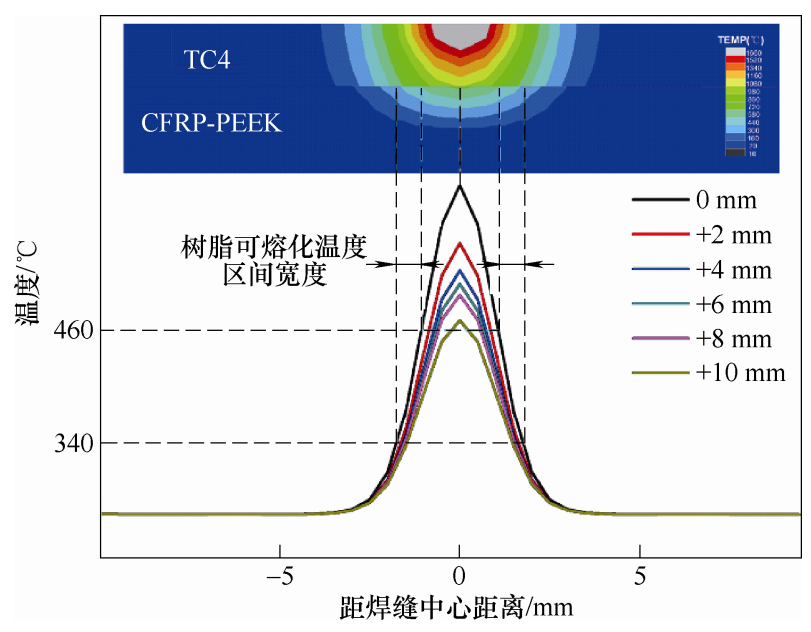

图 10 不同离焦量下 CFRP-PEEK 界面温度分布曲线

以上虽对连接温度场进行了模拟, 且与实际值 较为接近, 但是在试验时, 由于聚合物在红外线波 段是透明的，无法采用红外摄像机对其温度变化进 行测量, 而且也很难精确将热电偶放置于连接界面 
处, 因此获得实际激光与材料的热作用过程较为困 难。针对此问题, 学者 LAMBIASE 等 ${ }^{[32]}$ 建立了一 个校准和验证有限元模型的程序, 并通过适当的简 化采用了常用且相对便宜的仪器包括功率计、红外 摄像机等对程序进行了验证, 分别对聚合物的吸收 率、透射率以及反射率和金属材料对激光的反射率 进行了测量, 最终获得了连接温度场的分布情况。

图 11 为对聚碳酸酯(Polycarbonate, PC)与 AISI304 进行激光连接时实际接头与模拟温度场的对比结 果 ${ }^{[32]}$, 可以发现该模型具有一定的准确性。

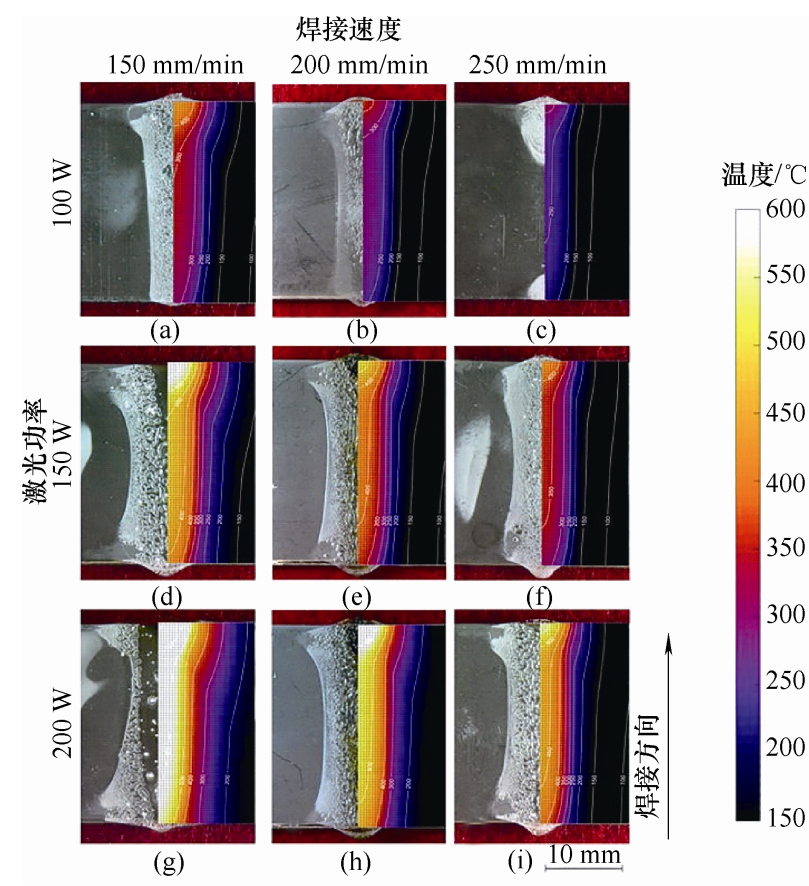

图 11 聚碳酸酯与 AISI304 进行激光连接实际接头与模拟 温度场的对比结果(左: 实际接头, 右: 模拟结果)

\section{3 气泡产生及抑制}

对于金属与塑料的连接, 气泡的产生及其分布 对接头的强度具有重要影响。日本长冈技术科学大 学 MIYASHITA 等 ${ }^{[33]}$ 在 304 不锈钢与 PET 和 PC 的 激光连接过程中, 发现在连接界面处随着热输入的 增加产生了不同数量和尺寸的气泡, 合适的气泡尺 寸被认为可产生高压使得熔融的塑料与不锈钢紧密 结合。当气泡超过一定尺寸时, 接头的接触面积减 小, 从而使接头强度降低。埃及中央治金研究与发 展研究所 WAHBA 等 ${ }^{[34]}$ 利用高功率二极管激光器对 镁合金与 PET 分别进行了激光热导焊和透射焊, 发 现热导焊的接头强度大于透射焊的强度, 分析其原 因是两种焊接方式中气泡的形态有所差异, 热导焊 中接头界面产生离散分布的气泡形态, 比透射焊中 界面产生的网孔状气泡更有利于金属与塑料的连
接。日本大阪大学 JUNG 等 ${ }^{[24]}$ 通过 $\mathrm{Q}$ 质谱仪对 CFRP 与不锈钢的接头中塑料熔化区产生的亚毫米级气泡 进行了检测, 结果显示该气泡内部的化学成分包括 CFRP 热解产生的氢气及碳氢化合物系列, 同时还 有空气中的氮气, 这解释了气泡产生的来源及起因。

2015 年, 清华大学 TAN 等 ${ }^{[35]}$ 对 CFRP 与低碳 钢激光热传导过程中气泡的形成机理进行了研究。 他们根据气孔的形貌及分布特征将其分为两类: I 型气孔和 II 型气孔, 如图 12 所示 ${ }^{[35]}$ 。其中, I 型 气孔只有当热输入大于 $77.8 \mathrm{~J} / \mathrm{mm}$ 时才会出现, 内壁 光滑, 分布在熔区中心部位的碳纤维与钢的结合界面 附近, 主要是由碳纤维热解产生的气体产物如二氧化 碳、氨气、水和碳氢化合物等引起的; 而 II 型气孔在 所有条件下都会产生, 内部粗糙, 分布在最终凝固的 区域，是由凝固阶段熔化的 CFRP 收缩产生。

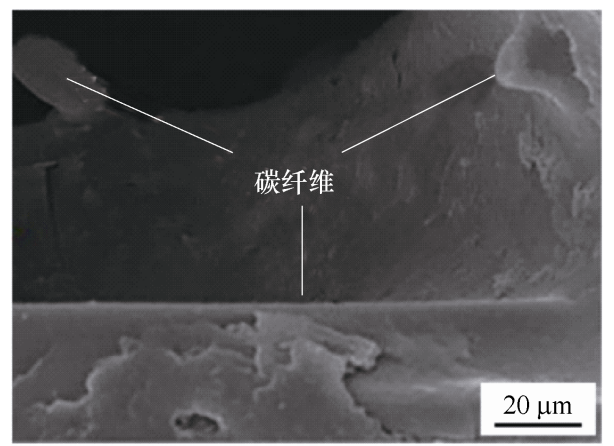

(a) I型气孔

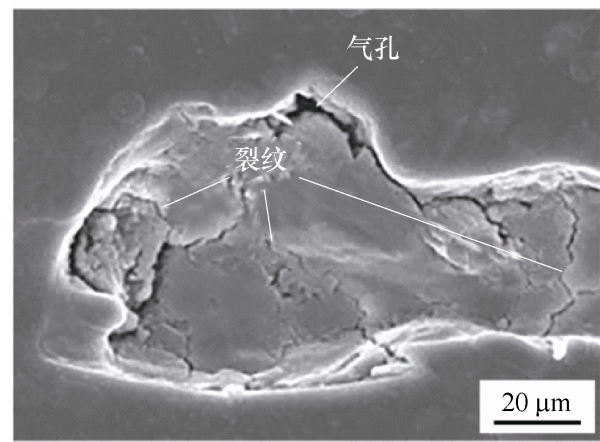

(b) II型气孔

图 12 两种类型气孔形貌特征

由此得出, 气泡的产生不可避免, 而不同形貌 及大小的分布则会对强度具有不同的作用。大部分 的研究中, 气泡被作为解释强度的原因, 而很少对 其进行抑制。香港理工大学 $\mathrm{CHEN}^{\text {等 }}{ }^{[3,36}$ 提出了一 种超声振动辅助下的金属与塑料激光连接的新方 法, 如图 13 所示 ${ }^{[3]}$ 。他们发现在超声振动下对纯 $\mathrm{Ti}$ 与 PET 进行激光连接, 其接头处气泡数量可有效 减少, 从而促进了金属与塑料的化学结合, 最终极 大地提高了接头强度。此外, 清华大学 ZHANG 等 ${ }^{[19]}$ 利用高速激光毛化技术在低碳钢表面制备了 不同尺寸和密度的凸起结构, 与 CFRP 进行激光连 
接, 发现具有这种凸起结构的表面形貌可改变热过 程, 使得气孔率由 $7.13 \%$ 降低到 $1.26 \%$, 而接头强 度由 $9.3 \mathrm{MPa}$ 提高到 $30.8 \mathrm{MPa}$ 。

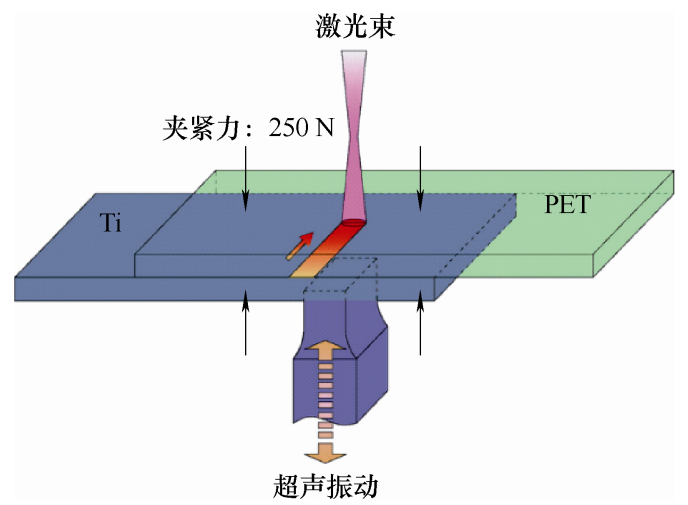

图 13 超声辅助金属与塑料激光连接示意图

\section{4 接头性能调控}

金属与塑料的连接界面位置主要有三种力的作 用: 机械连接(机械针固力)、物理连接(范德瓦尔华力, 粘附力)和化学键合(化学力)。通过对 304 不锈钢与 PET 激光透射焊的界面进行观察, KATAYAMA 等 ${ }^{[10]}$ 对其连接机理进行了解释。作者发现, 在塑料熔化产 生气泡的压作用下，金属与塑料发生物理接触, 且熔 融的塑料进入金属表面的不平整凹坑, 形成机械嵌合 (机械针固效应)。利用 XPS 和 TEM 证明了界面产生 了新的化学键, 实现了二者的紧密结合。图 $14 \mathrm{a}$ 和 $14 \mathrm{~b}$ 分别为 304 与 PET 的界面 SEM 及 TEM 结果 ${ }^{[10]}$ 。

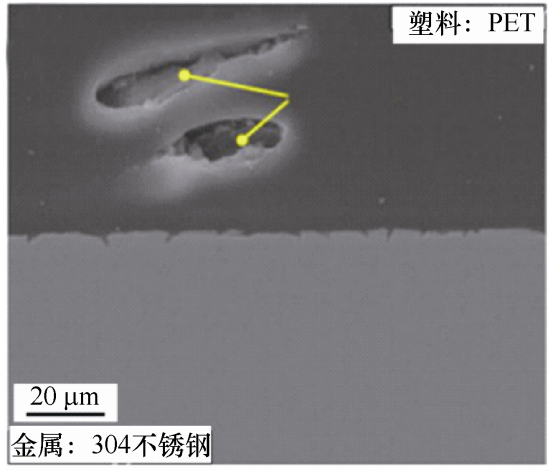

(a) 接头界面SEM形貌

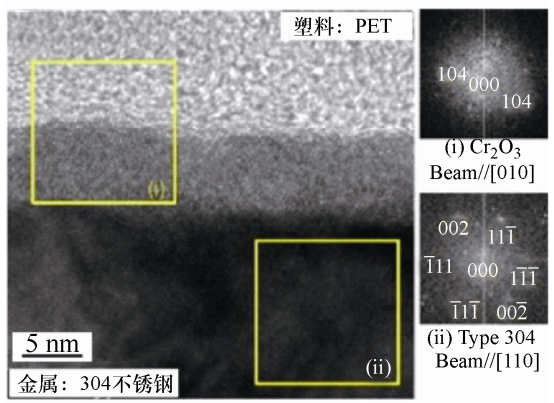

(b) 接头界面TEM形貌

图 14304 不锈钢/PET 接头界面 SEM 和 TEM 形貌
基于金属与塑料的连接机理，学者们对接头性 能进行了调控。调控方式主要是加强机械针固力从 而提高接头强度。作为一种高效率、可控制、精度 高的表面处理技术, 激光表面微织构技术(激光毛化) 已被应用于金属与塑料连接的强度调控。其原理是 利用激光烧蚀作用在金属表面预制具有一定深度的 凹坑或网格等形貌的微织构, 使得熔融塑料融入微织 构中，与其形成机械咬合，同时增加二者接触面积， 从而提高接头强度。德国弗郎霍夫激光技术研究所 ROESNER 等 ${ }^{[22]}$ 利用微秒激光器在 304 不锈钢表面加 工出宽 $40 \mu \mathrm{m}$ 、深 $50 \mu \mathrm{m}$ 的凹坑结构, 分别与 PC、 PA66、GFPA66 进行了激光连接，发现其连接接头的 最高剪切强度可达到 $24 \mathrm{MPa}$ 。在此基础上，德国巴 伐利亚激光中心有限公司 AMEND 等 ${ }^{[37]}$ 在 5182 铝合 金表面制备了不同深度的凹坑和凹槽结构, 分别与三 种塑料进行了激光连接。研究发现随着深度的增加, 其强度有所增加，但深度过大时对强度提高没有显著 作用。作者发现，这种深度较大的结构虽然提高了接 触面积，但因熔融塑料有限，无法实现对金属表面的 充分结合，从而产生不利效果。图 15 为具有凹坑结 构的接头界面, 发现塑料无法进入较深结构, 因此产 生了空气间隙，降低了强度 ${ }^{[37]}$ 。

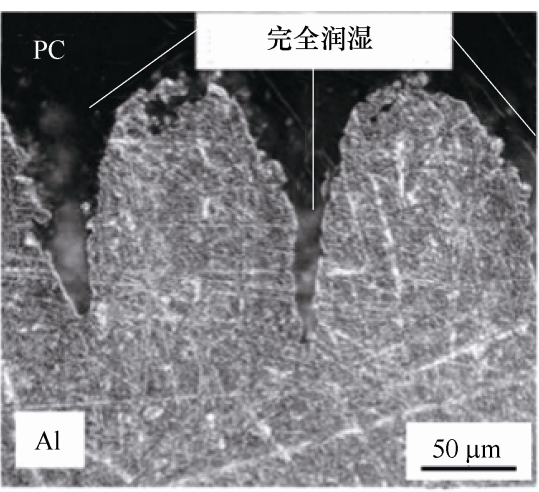

(a) 铝合金表面浅凹坑结构接头形貌

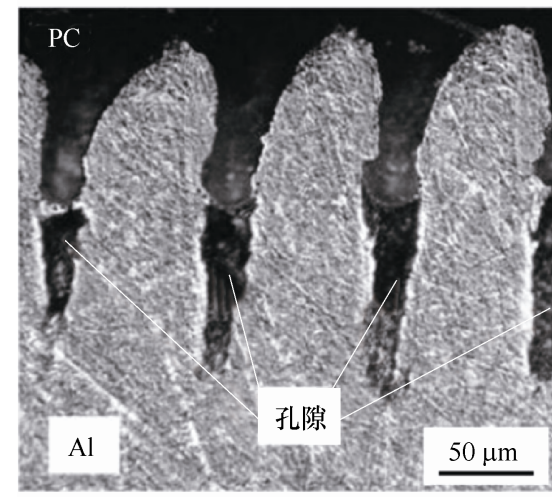

(b) 铝合金表面深凹坑结构接头形貌

图 15 凹坑结构中的界面结合情况

中国科学院宁波材料技术与工程研究所 JIAO 等 ${ }^{[38]}$ 在 A7075 铝合金表面制备了不同间距的网格微织 构, 并与 CFPA6 进行了激光热导焊及接头性能的调 
控。试验发现, 间距为 $0.2 \mathrm{~mm}$ 时, 机械针固效应最 显著, 强度获得最大值为 $37.5 \mathrm{MPa}$, 比未作处理的 接头强度提高了 $260 \%$ 。而当间距进一步减小时, 由 于热积累作用更加明显, 破坏了网格微织构的表面 形貌, 并因此减小了金属与塑料的接触面积, 降低 了金属基体与塑料的结合力，同样当间距过大时接 触面积逐渐减小，仍不具有较高的机械针固作用。 西班牙的 RODRÍGUEZ-VIDAL 等 ${ }^{[39]}$ 研究了具有不同 深宽比微织构对 HC420 钢与 GFPA6 激光透射焊连接 强度的影响, 试验发现深宽比在一定范围内可提高 接头的强度, 超过限值之后影响不大。笔者所在研 究团队采用纳秒激光器也实现了如图 16 所示的钛合 金、铝合金及 304 不锈钢表面微织构的制备, 并建 立不同材料表面激光毛化处理工艺参数与微织构深

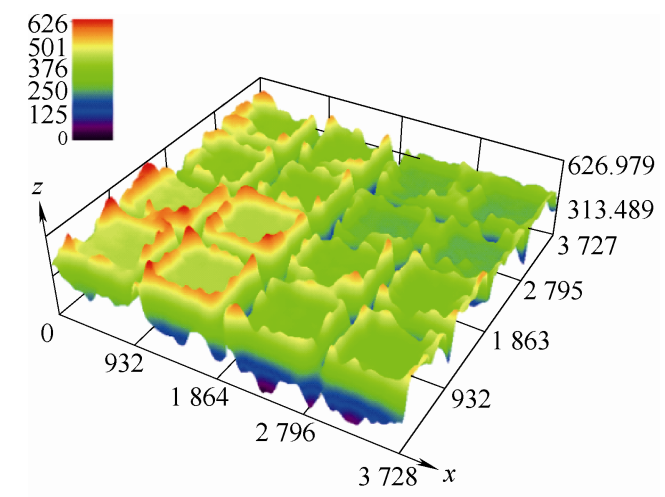

(a) 钛合金表面微织构加工形貌

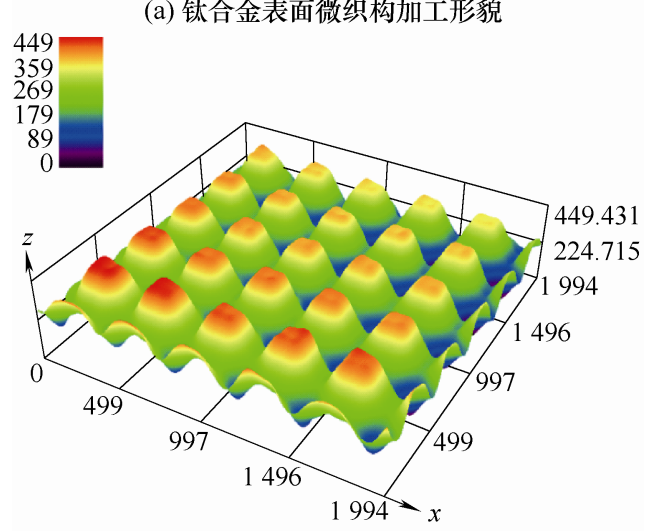

(b) 铝合金表面微织构加工形貌

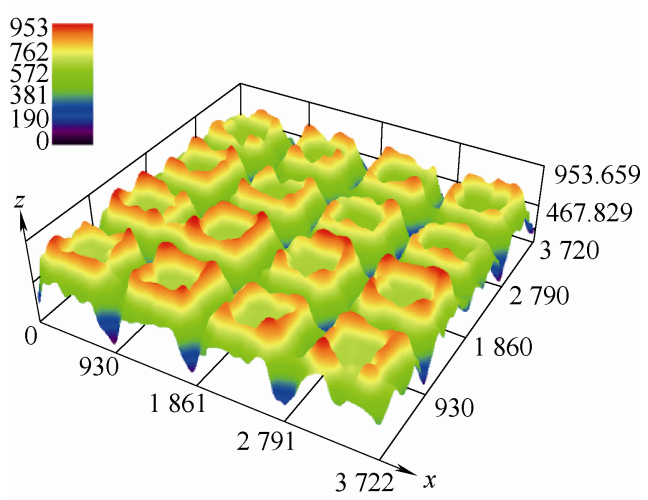

(c) 304不锈钢表面微织构加工形貌

图 16 钛合金、铝合金及 304 不锈钢表面微织构形貌
宽比的对应关系。材料表面微织构深宽比的变化对 连接强度及连接机理的影响将在后续研究中开展。

清华大学 ZHANG 等 ${ }^{[18]}$ 利用高速激光毛化技术 在 A7050 铝合金表面制备了不同密度的凸起结构, 并与 CFPA6 进行了激光热传导焊。试验发现随着凸 起结构密度的增加, 接头强度不断增加, 最高剪切 强度为 $39.0 \mathrm{MPa}$, 与未作处理的接头相比提高了 4 倍。作者认为, 凸起结构的机械嵌合作用较微织构 的效果更显著, 这主要归因于凸起结构可产生更强 的钉扎作用。同时, 他们也对金属与塑料之间化学 力的提高展开了研究 ${ }^{[40]}$, 对 A6061 铝合金表面进行 了如图 17 所示的不同时长阳极氧化处理后与 CFPA6 进行了连接, 发现在不同氧化时间处理下, 接头强 度均有所提高, 最高剪切强度达到 $41.8 \mathrm{MPa}$, 与未 作处理的接头相比提高了 8 倍。分析认为, 在铝合 金表面形成的纳米多孔结构使得铝合金与塑料的界 面产生过渡层, 并形成图 18 所示 ${ }^{\left[{ }^{[0]}\right.}$ 的新化学键 “Al-O-PA6”。为提高低碳钢与 CFPA6 的连接强度, 清华大学的谭向虎等 ${ }^{[41]}$ 在低碳表面电镀一定厚度的 $\mathrm{Cr}$ 层, 结果发现连接界面形成 Cr-O-PA6T 化学键合 使得接头强度由未作处理的 $9.32 \mathrm{MPa}$ 提高到 22.14 $\mathrm{MPa}$ 。另外, ARAI 等 ${ }^{[42]}$ 利用紫外线照射 $\mathrm{COP}$ (环烯 烃聚合物)表面, 增加了表面官能团, 自由能随之提 高, 这使得其与 304 不锈钢之间激光连接的粘附力 得到提升, 剪切强度从 $0 \mathrm{MPa}$ 提高到 $8 \mathrm{MPa}$ 。

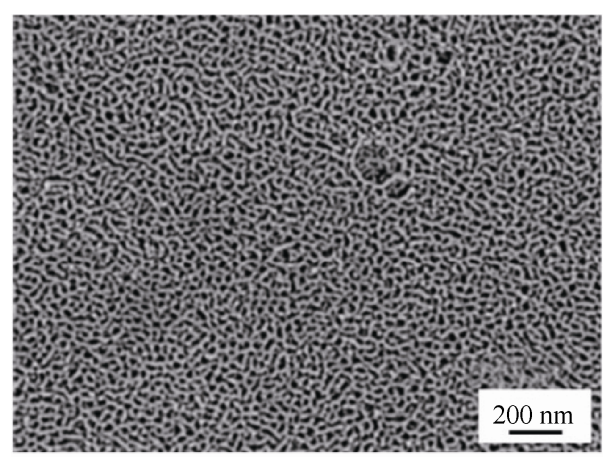

(a) 阳极氧化 $5 \mathrm{~min}$ 后A6061表面形貌

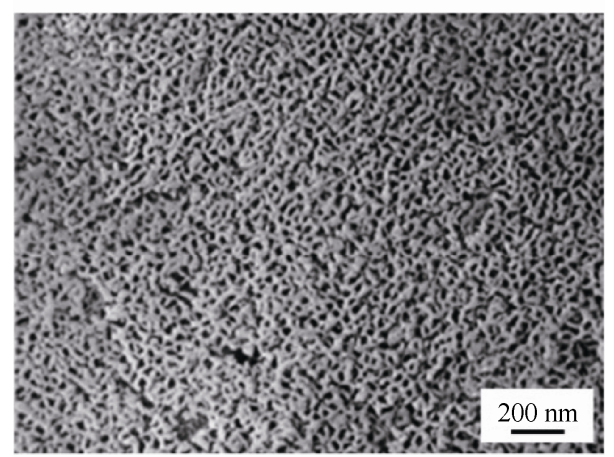

(b) 阳极氧化10 min后A6061表面形貌

图 17 不同时长阳极氧化处理后 A6061 表面形貌 


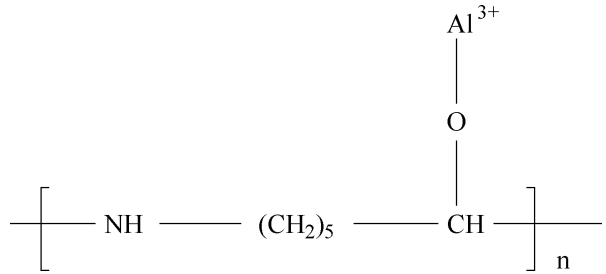

图 18 阳极氧化下 $\mathrm{Al} / \mathrm{CFRP}$ 生成的化学键

\section{5 展望}

金属与塑料的激光连接作为满足工业轻量化发 展的重要途径，针对金属/塑料间特殊的性质差异， 通过多种连接工艺手段对接头性能进行调控使金 属/塑料实现了可靠连接。目前, 关于金属与塑料激 光连接的研究内容涉及工艺研究、连接界面温度场 仿真模拟、界面位置气泡产生及抑制和接头性能调 控等多个方面。

但是金属与塑料激光连接技术仍然存在许多问 题亟待解决。

(1) 目前多数研究主要针对不锈钢及铝合金与 塑料的激光连接工艺展开, 而针对钛合金、镁合金 等轻质合金研究相对较少。

(2) 目前对金属与单一聚合物的激光连接的研 究相对较多, 而纤维增强热塑复合材料因具有更高 强的使用性能，与金属的连接将具有良好的发展前 景。金属与纤维增强热塑复合材料的研究还处于初 步阶段，虽在工艺方法，表面处理手段等方面该方 向的连接已取得了一定的进展, 但增强纤维在界面 连接过程中的作用及连接强度的影响尚不明确。

(3) 激光连接过程中，界面位置受热传导及局 部过热影响而产生的气泡作为连接强度的重要影响 因素，目前只针对气泡类型及产生机理展开了相关 研究, 对于如何控制气泡的形态及分布以提高连接 强度仍需进一步研究。

(4) 连接机理方面, 对于金属与塑料连接界面 位置机械针固及物理连接相对较多，不同材料间界 面位置化学键的形成机理仍需进一步明确。上述问 题的解决将有助于金属/塑料激光焊接工艺研究及 性能调控的进一步发展。

\section{参 考 文 献}

[1] MARTOWIBOWO S Y, KASWADI A. Optimization and simulation of plastic pnjection process using genetic algorithm and moldflow $[\mathrm{J}]$. Chinese Journal of Mechanical Engineering, 2017, 30(2): 398-406.

[2] WU S, GAO D, LIANG Y, et al. Experimental study on influence of dimples on lubrication performance of glass fiber-epoxy resin composite under natural seawater lubrication[J]. Chinese Journal of Mechanical Engineering, 2017, 30(1): 110-117.

[3] CHEN Y J, YUE T M, GUO Z N. A new laser joining technology for direct-bonding of metals and plastics[J]. Materials \& Design, 2016, 110: 775-781.

[4] LAMBIASE F, GENNA S. Laser-assisted direct joining of AISI304 stainless steel with polycarbonate sheets: Thermal analysis, mechanical characterization, and bonds morphology[J]. Optics \& Laser Technology, 2017, 88: 205-214.

[5] SAIRAJAN K K, AGLIETTI G S, MANI K M. A review of multifunctional structure technology for aerospace applications[J]. Acta Astronautica, 2016, 120: 30-42.

[6] 李喜平, 刘傅文, 王爽, 等. 金-塑微结构注射成型仿 真与试验研究 [J]. 机械工程学报, 2016, 52(22): 61-69. LI Xiping, LIU Fuwen, WANG Shuang, et al. Simulation and experiment study of metal-polymer composite injected with microstructure[J]. Journal of Mechanical Engineering, 2016, 52(22): 61-69.

[7] KARSLI N G, AYTAC A. Tensile and thermomechanical properties of short carbon fiber reinforced polyamide 6 composites[J]. Composites Part B: Engineering, 2013, 51: 270-275.

[8] PRAMANIK A, BASAK AK, DONG Y, et al. Joining of carbon fiber reinforced polymer (CFRP) composites and aluminium alloys-A review[J]. Composites Part A: Applied Science and Manufacturing, 2017, 101: 1-29.

[9] ZUO Y, CAO Z, CAO Y, et al. Dynamic behavior of $\mathrm{CFRP} / \mathrm{Ti}$ single-lap pinned joints under longitudinal electromagnetic dynamic loading $[\mathrm{J}]$. Composite Structures, 2018, 184: 362-371.

[10] KATAYAMA S, KAWAHITO Y. Laser direct joining of metal and plastic[J]. Scripta Materialia, 2008, 59: $1247-1250$

[11] 甄立冬, 王妍. 热塑铆接工艺研究 $[\mathrm{C} / \mathrm{CD}] / /$ 中国电子学 会生产技术学分会机械加工专业委员会学术年会. 1998.

ZHEN Lidong, WANG Yan. Research on thermoplastic riveting process $[\mathrm{C} / \mathrm{CD}] / /$ Annual Meeting of the Professional Committee of Mechanical Processing, Society of Production Technology, China Electronics Society. 1998.

[12] RETIZ V, MEINHARD D, RUCK S, et al. A comparison of IR- and UV-laser pretreatment to increase the bonding strength of adhesively joined aluminum/CFRP components[J]. Composites Part A: Applied Science and Manufacturing, 2017, 96: 18-27.

[13] 靳少龙. 高分子材料 ABS 与金属电阻焊连接工艺及机 
理的研究[D]. 兰州: 兰州理工大学, 2017 .

JIN Shaolong. Study on the joining process and mechanism of polymer ABS and metal resistance welding [D]. Lanzhou: Lanzhou University of Technology, 2017.

[14] 田英超, 李五一, 张智勇, 等. 含 $\mathrm{Ti}$ 钎料钎焊碳纤维 复合材料与钛合金的界面微观分析 [J]. 航天制造技术, 2009(4): 17-20.

TIAN Yingchao, LI Wuyi, ZHANG Zhiyong, et al. Microscopic analysis of interface between carbon fibre composite and titanium alloy brazed with Ti brazing filler metal[J]. Aerospace Manufacturing Technology, 2009(4): $17-20$.

[15] LIONETTO F, MELE C, LEO P, et al. Ultrasonic spot welding of carbon fiber reinforced epoxy composites to aluminum[J]. Mechanical and Electrochemical Characterization. Composites Part B: Engineering, 2018, 144: 134-142.

[16] 谢一鸣. FRTP 同种及 FRTP 与铝合金异种材料搅拌摩 擦焊[D]. 南昌: 南昌航空大学, 2012.

XIE Yiming. Friction stir welding of FRTP homologous and FRTP dissimilar materials with aluminium alloy[D]. Nanchang: Nanchang Aeronautical University, 2012.

[17] 王晓虹, 谷晓燕, 孙大千. 钢/铝异种金属激光焊接头 界面特性的研究 [J]. 机械工程学报, 2017(4): 26-33. WANG Xiaohong, GU Xiaoyan, SUN Daqian. Research on interface characteristic of laser welding joints of steel/aluminum dissimilar materials[J]. Journal of Mechanical Engineering, 2017(4): 26-33.

[18] ZHANG Z, SHAN J, TAN X, et al. Improvement of the laser joining of CFRP and aluminum via laser pre-treatment $[\mathrm{J}]$. The International Journal of Advanced Manufacturing Technology, 2016, 90: 3465-3472.

[19] ZHANG Z, TAN X, ZHANG J, et al. Suppression of shrinkage porosity in laser-joining of CFRP and steel using a laser surface modification process "Surfi-Sculpt (®) [J]. International Journal of Adhesion and Adhesives, 2018, 85: 184-192.

[20] 郑怀中. 304 不锈钢与聚对苯二甲酸乙二醇酯(PET)异 种材料的焊接工艺研究[D]. 镇江: 江苏大学, 2017 .

ZHENG Huaizhong. Welding technology of 304 stainless steel and polyethylene terephthalate (PET) dissimilar material [D]. Zhenjiang: Jiangsu University, 2017.

[21］孙雪会. 镁合金与聚乙烯对苯二甲酸酯(PET)焊接工艺 研究[D]. 大连: 大连理工大学, 2014.

SUN Xuehui. Welding technology of magnesium alloy and polyethylene terephthalate (PET) [D]. Dalian: Dalian University of Technology, 2014.

[22] ROESNER A, SCHEIK S, OLOWINSKY A, et al. Laser assisted joining of plastic metal hybrids[J]. Physics
Procedia, 2011, 12: 370-377.

[23] JUNG K W, KAWAHITO Y, TAKAHASHI M, et al. Laser direct joining of carbon fiber reinforced plastic to zinc-coated steel[J]. Materials \& Design, 2013, 47 : 179-188

[24] JUNG K W, KAWAHITO Y, KATAYAMA S. Laser direct joining of carbon fibre reinforced plastic to stainless steel[J]. Science and Technology of Welding and Joining, 2013, 16: 676-680.

[25] JUNG K W, KAWAHITO Y, TAKAHASHI M, et al. Laser direct joining of carbon fiber reinforced plastic to aluminum alloy[J]. Journal of Laser Applications, 2013, 47: $179-188$.

[26] SHENG L Y, JIAO J K, LAI C. Assessment of the microstructure and mechanical properties of a laser-joined carbon fiber-reinforced thermosetting plastic and stainless steel[J]. Strength of Materials, 2018， 50: 752-763.

[27] HUSSEIN F I, AKMAN E, OZTOPRAK B G, et al. Evaluation of PMMA joining to stainless steel 304 using pulsed Nd: YAG laser[J]. Optics \& Laser Technology, 2013, 49: 143-152.

[28] 黄创. 激光直接连接金属与聚合物的试验研究及数值 模拟[D]. 镇江: 江苏大学, 2014.

HUANG Chuang. Experimental study and numerical simulation of laser direct bonding of metal and polymer [D]. Zhenjiang: Jiangsu University, 2014.

[29] 黄怡洁. 不锈钢(304)与聚甲基丙烯酸甲酯(PMMA)异 种材料激光焊接试验研究 [D]. 广州: 广东工业大学, 2018.

HUANG Yijie. Laser welding of stainless steel (304) and polymethyl methacrylate (PMMA) dissimilar materials [D]. Guangzhou: Guangdong University of Technology, 2018.

[30] HUSSEIN F I, SALLOOMI K N, AKMAN E, et al. Finite element thermal analysis for PMMA/st.st.304 laser direct joining[J]. Optics and Laser Technology, 2017, 87: 64-71.

[31] 王强. 碳纤热塑复合材料/不锈钢激光连接实验与数值 模拟研究[D]. 宁波: 宁波大学, 2017.

WANG Qiang. Experimental and numerical simulation of laser bonding of carbon fiber thermoplastic composites/ stainless steel [D]. Ningbo: Ningbo University, 2017.

[32] LAMBIASE F, GENNA S, KANT R. A procedure for calibration and validation of $\mathrm{FE}$ modelling of laser-assisted metal to polymer direct joining[J]. Optics \& Laser Technology, 2018, 98: 363-372.

[33] MIYASHITA Y, TAKAHASHI M, TAKEMI M, et al. Dissimilar materialsmicro welding between stainless steel and plastics by using pulse YAG laser[J]. Journal of Solid Mechanics \& Materials Engineering, 2009, 3: 409-415. 
[34] WAHBA M, KAWAHITO Y, KATAYAMA S. Laser direct joining of AZ91D thixomolded Mg alloy and amorphous polyethylene terephthalate[J]. Journal of Materials Processing Technology, 2011, 211: 1166-1174.

[35] TAN X, ZHANG J, SHAN J, et al. Characteristics and formation mechanism of porosities in CFRP during laser joining of CFRP and steel[J]. Composites Part B : Engineering, 2015, 70: 35-43.

[36] CHEN Y J, YUE T M, GUO Z N. Laser joining of metals to plastics with ultrasonic vibration[J]. Journal of Materials Processing Technology, 2017, 249: 441-451.

[37] AMEND P, PFINDEL S, SCHMIDT M. Thermal joining of thermoplastic metal hybrids by means of mono- and polychromatic radiation[J]. Physics Procedia, 2013, 41: 98-105.

[38] JIAO J, JIA S, XU Z, et al. Laser direct joining of CFRTP and aluminium alloy with a hybrid surface pre-treating method $[\mathrm{J}]$. Composites Part B: Engineering, 2019, 173 : 106911.

[39] RODRÍGUEZ-VIDAL E, SANZ C, LAMBARRI J, et al. Experimental investigation into metal micro-patterning by laser on polymer-metal hybrid joining[J]. Optics \& Laser Technology, 2018, 104: 73-82.

[40] ZHANG Z, SHAN J G, TAN X H, et al. Effect of anodizing pretreatment on laser joining CFRP to aluminum alloy A6061[J]. International Journal of Adhesion and Adhesives, 2016, 70: 142-151.

[41] 谭向虎, 单际国, 任家烈. 镀 Cr 层对低碳钢 / CFRP 激光连接接头剪切强度及界面结合特征的影响 [J]. 金 属学报, 2013, 49(6): 751-756.

TAN Xianghu, SHAN Jiguo, REN Jialie. Effect of $\mathrm{Cr}$ plating on shear strength and interfacial bonding characteristics of low carbon steel/CFRP laser bonded joints [J]. Journal of Metals， 2013， 49(6): 751-756.

[42] ARAI S, KAWAHITO Y, KATAYAMA S. Effect of surface modification on laser direct joining of cyclic olefin polymer and stainless steel[J]. Materials \& Design, 2014, 59: 448-453.

作者简介: 檀财旺, 男, 1986 年出生, 博士, 副教授, 硕士研究生导 师。主要研究方向为轻量化激光连接、激光-电弧复合焊、能场辅助激 光焊接等。

E-mail: tancaiwang@163.com 